Honam Mathematical J. 34 (2012), No. 3, pp. 423-438

http://dx.doi.org/10.5831/HMJ.2012.34.3.423

\title{
COUNTING PROBLEMS IN GENERALIZED PAPER FOLDING SEQUENCES
}

\author{
JungheE YUN ${ }^{\dagger}$ AND NAHMWOO HAHM ${ }^{\ddagger}$
}

\begin{abstract}
In this paper, we discuss numbers of downwards and upwards in generalized paper folding sequences. We compute the exact number of downwards and upwards in $R_{p}^{n}$ and $\left(R_{p} R_{q}\right)^{n}$ by using the properties of recursive sequences where $n, p$ and $q$ are natural numbers with $p \geq 2$ and $q \geq 2$.
\end{abstract}

\section{Introduction and Preliminaries}

When we fold a sheet of paper and unfold it, the paper has some creases. Dekking [4] used 0 for a crease that makes the paper upward and 1 for a crease that makes the paper downward. Note that a paper folding sequence is the sequence of $0 \mathrm{~s}$ and $1 \mathrm{~s}$ obtained by unfolding a sheet of paper which has been folded many times.

Paper folding sequences have been studied extensively by Allouche, Bates, Bunder, Tognetti, France and Poorten in $[1,2,5]$ since Davis and Knuth introduced its concept in [3]. Dekking [4] showed how the automatic structure of the paper folding sequences lead to self-similarity of the curves. Lee, Kim and Choi [6] showed the trace of paper folding sequences using $(0,1)$ codes and $(0,1)$ matrices. In this paper, we introduce generalized paper folding sequences and compute the exact number of $0 \mathrm{~s}$ and $1 \mathrm{~s}$ in generalized paper folding sequences.

Received July 25, 2012. Accepted August 27, 2012.

2000 Mathematics Subject Classification. 97A20, 68R99.

Key words and phrases. paper folding sequence, counting problem, upward, downward.

† Sooji Hwang, Jinrae Kim, Sunggye Lee, Chansu Park, Jeongyin Park(2nd grade) and Junhwi Lim(1st grade) at Incheon Science High School are collaborators of this research.

$\dagger$ This research was supported by 2012 government funds(the Science and Technology Promotion Fund / the Lottery Fund) with the assistance of the Korea Foundation for the Advancement of Science and Creativity.

$\ddagger$ Corresponding author. 
When we fold a sheet of paper, we may fold it left over right or right over left. We use $R$ when we fold a sheet of paper left over right and $L$ when we fold a sheet of paper right over left. When we fold a sheet of paper left over right and rotate it $180^{\circ}$ angles, the creases are the same as that of the paper folding right over left.

Let $p, q, n \in \mathbb{N}$ with $p \geq 2$ and $q \geq 2$. If we fold a sheet of paper in $p$ left over right, we get a generalized paper folding sequence and denote it by $R_{p}$. If we iterate $R_{p}$ process $n$ times, then we get another generalized paper folding sequence and denote it by $R_{p}^{n}$. Similarly, if we fold a sheet of paper in $p$ left over right and then fold the result in $q$ left over right, we get a paper folding sequence and denote it by $R_{p} R_{q}$. If we iterate $R_{p} R_{q}$ process $n$ times, then we get another generalized paper folding sequence and denote it by $\left(R_{p} R_{q}\right)^{n}$.

Example 1.1. Some examples of generalized paper folding sequences are given as follows :

(1) $R_{4}^{3}: \begin{array}{lllllllllllllllllllllllllll} & 0 & 0 & 0 & 0 & 1 & 1 & 1 & 0 & 0 & 0 & 0 & 0 & 1 & 1 & 1 & 1 & 0 & 0 & 0 & 1 & 1 & 1 & 1 & 1 & 0 & 0\end{array}$

(2) $\left(R_{2} R_{3}\right)^{2} \quad: \quad \begin{array}{llllllllllllllllllllllll}0 & 0 & 0 & 1 & 1 & 0 & 0 & 0 & 1 & 1 & 1 & 0 & 0 & 0 & 0 & 1 & 1 & 1 & 0 & 0 & 1 & 1 & 1 & 1\end{array}$ $\begin{array}{lllllllllllll}0 & 0 & 0 & 1 & 1 & 1 & 0 & 0 & 1 & 1 & 1\end{array}$

(3) $\left(R_{3} R_{2}\right)^{2} \quad: \quad \begin{array}{llllllllllllllllllllllll}0 & 0 & 1 & 0 & 0 & 1 & 1 & 1 & 0 & 1 & 1 & 1 & 0 & 0 & 1 & 0 & 0 & 0 & 1 & 1 & 0 & 1 & 1 & 1\end{array}$ $\begin{array}{lllllllllllll}0 & 0 & 1 & 0 & 0 & 1 & 1 & 1 & 0 & 1 & 1\end{array}$

Let $X$ be a paper folding sequence. We define $X^{c}$ the paper folding sequence obtained by reversing the order and swapping 0 s and $1 \mathrm{~s}$ in $X$. $|X|$ denotes the number of all 0 s and 1 s in $X .|X|_{0}$ and $|X|_{1}$ denote the number of all 0s in $X$ and all $1 \mathrm{~s}$ in $X$, respectively. The following lemma can be easily obtained by the definitions of $|X|,|X|_{0},|X|_{1}$ and $X^{c}$.

Lemma 1.2. Let $X$ be a paper folding sequence. Then we have

(1) $|X|=|X|_{0}+|X|_{1}$

(2) $\left|X^{c}\right|_{0}=|X|_{1}$

(3) $\left|X^{c}\right|_{1}=|X|_{0}$

(4) $\left|X^{c}\right|=|X|$. 


\section{Number of $0 \mathrm{~s}$ and $1 \mathrm{~s}$ in $R_{p}^{n}$}

Davis and Knuth [3] proved the following theorem and it provided us with impetus to probe the problems related to number of downwards and upwards in generalized paper folding sequences.

Theorem 2.1. Let $p \in \mathbb{N}$ with $p \geq 2$. If $R_{p}$ and $X$ are paper folding sequences, then

$$
R_{p} X= \begin{cases}\left(X^{c} 1 X 1 X^{c} 1 X 1 \cdots 1 X^{c} 1 X\right) & \text { if } p \text { is even } \\ \left(X 1 X^{c} 1 X 1 X^{c} 1 \cdots 1 X^{c} 1 X\right) & \text { if } p \text { is odd } .\end{cases}
$$

First, we compute the number of $0 \mathrm{~s}$ and $1 \mathrm{~s}$ in $R_{p}^{n}$ using Theorem 2.1 and the properties of a recursive sequence.

Theorem 2.2. If $p$ is an even number with $p \geq 2$ and $n \in \mathbb{N}$, then

$$
\left|R_{p}^{n}\right|_{0}=\frac{1}{2}\left(p^{n}-p\right) \quad \text { and } \quad\left|R_{p}^{n}\right|_{1}=\frac{1}{2}\left(p^{n}+p-2\right) .
$$

Proof. Since $p$ is even and $R_{p}^{n}=R_{p} R_{p}^{n-1}$, Theorem 2.1 gives

$$
R_{p}^{n}=\left(\left(R_{p}^{n-1}\right)^{c} 1 R_{p}^{n-1} 1 \cdots 1\left(R_{p}^{n-1}\right)^{c} 1 R_{p}^{n-1}\right) .
$$

Note that $\left(R_{p}^{n-1}\right)^{c}$ and $R_{p}^{n-1}$ appear $\frac{p}{2}$ times and $\frac{p}{2}$ times in (2.3), respectively. In addition, 1 appears $p-1$ times in (2.3).

By (2.3) and Lemma 1.2, we have

$$
\begin{aligned}
\left|R_{p}^{n}\right| & =\frac{p}{2}\left|\left(R_{p}^{n-1}\right)^{c}\right|+\frac{p}{2}\left|R_{p}^{n-1}\right|+(p-1) \\
& =\frac{p}{2}\left|R_{p}^{n-1}\right|+\frac{p}{2}\left|R_{p}^{n-1}\right|+(p-1) \\
& =p\left|R_{p}^{n-1}\right|+(p-1) .
\end{aligned}
$$

By adding 1 on both sides of (2.4), we get

$$
\begin{aligned}
\left|R_{p}^{n}\right|+1 & =p\left|R_{p}^{n-1}\right|+p \\
& =p\left(\left|R_{p}^{n-1}\right|+1\right) \\
& =p^{2}\left(\left|R_{p}^{n-2}\right|+1\right) \\
& =\cdots \\
& =p^{n}\left(\left|R_{p}^{0}\right|+1\right) \\
& =p^{n},
\end{aligned}
$$

since $\left|R_{p}^{0}\right|=0$. Thus

$$
\left|R_{p}^{n}\right|=p^{n}-1
$$


Now, we compute the number of $0 \mathrm{~s}$ in $R_{p}^{n}$. By (2.4), (2.6) and Lemma 1.2 , we get

$$
\begin{aligned}
\left|R_{p}^{n}\right|_{0} & =\frac{p}{2}\left|\left(R_{p}^{n-1}\right)^{c}\right|_{0}+\frac{p}{2}\left|R_{p}^{n-1}\right|_{0} \\
& =\frac{p}{2}\left|R_{p}^{n-1}\right|_{1}+\frac{p}{2}\left|R_{p}^{n-1}\right|_{0} \\
& =\frac{p}{2}\left|R_{p}^{n-1}\right| \\
& =\frac{p}{2}\left(p^{n-1}-1\right) \\
& =\frac{1}{2}\left(p^{n}-p\right) .
\end{aligned}
$$

Since the number of 1s can be computed by subtracting the number of 0 s from the total number of creases, we have

$$
\begin{aligned}
\left|R_{p}^{n}\right|_{1} & =\left|R_{p}^{n}\right|-\left|R_{p}^{n}\right|_{0} \\
& =\left(p^{n}-1\right)-\frac{1}{2}\left(p^{n}-p\right) \\
& =\frac{1}{2}\left(p^{n}+p-2\right) .
\end{aligned}
$$

Thus we complete the proof.

Now, we compute the number of $0 \mathrm{~s}$ and $1 \mathrm{~s}$ in $R_{p}^{n}$ when $p$ is odd with $p \geq 3$. In this case, we use a different property of a recursive sequence that is not used in Theorem 2.2.

Theorem 2.3. If $p$ is an odd number with $p \geq 3$ and $n \in \mathbb{N}$, then

$$
\left|R_{p}^{n}\right|_{0}=\frac{1}{2}\left(p^{n}-n p+n-1\right) \quad \text { and } \quad\left|R_{p}^{n}\right|_{1}=\frac{1}{2}\left(p^{n}+n p-n-1\right) .
$$

Proof. Since $p$ is odd and $R_{p}^{n}=R_{p} R_{p}^{n-1}$, Theorem 2.1 gives

$$
R_{p}^{n}=\left(R_{p}^{n-1} 1\left(R_{p}^{n-1}\right)^{c} 1 R_{p}^{n-1} 1 \cdots 1\left(R_{p}^{n-1}\right)^{c} 1 R_{p}^{n-1}\right) .
$$

Note that $\left(R_{p}^{n-1}\right)^{c}$ and $R_{p}^{n-1}$ appear $\frac{p-1}{2}$ times and $\frac{p+1}{2}$ times in (2.10), respectively. In addition, 1 appears $p-1$ times in (2.10).

By (2.10) and Lemma 1.2, we have

$$
\begin{aligned}
\left|R_{p}^{n}\right| & =\frac{p-1}{2}\left|\left(R_{p}^{n-1}\right)^{c}\right|+\frac{p+1}{2}\left|R_{p}^{n-1}\right|+(p-1) \\
& =\frac{p-1}{2}\left|R_{p}^{n-1}\right|+\frac{p+1}{2}\left|R_{p}^{n-1}\right|+(p-1) \\
& =p\left|R_{p}^{n-1}\right|+(p-1) .
\end{aligned}
$$


By adding 1 on both sides of (2.11), we get

$$
\begin{aligned}
\left|R_{p}^{n}\right|+1 & =p\left|R_{p}^{n-1}\right|+p \\
& =p\left(\left|R_{p}^{n-1}\right|+1\right) \\
& =p^{2}\left(\left|R_{p}^{n-2}\right|+1\right) \\
& =\cdots \\
& =p^{n}\left(\left|R_{p}^{0}\right|+1\right) \\
& =p^{n},
\end{aligned}
$$

since $\left|R_{p}^{0}\right|=0$. Thus

$$
\left|R_{p}^{n}\right|=p^{n}-1
$$

Now, we compute the number of $0 \mathrm{~s}$ in $R_{p}^{n}$. By (2.11), (2.13) and Lemma 1.2 , we get

$$
\begin{aligned}
\left|R_{p}^{n}\right|_{0} & =\frac{p-1}{2}\left|\left(R_{p}^{n-1}\right)^{c}\right|_{0}+\frac{p+1}{2}\left|R_{p}^{n-1}\right|_{0} \\
& =\frac{p-1}{2}\left|R_{p}^{n-1}\right|_{1}+\frac{p+1}{2}\left|R_{p}^{n-1}\right|_{0} \\
& =\left|R_{p}^{n-1}\right|_{0}+\frac{p-1}{2}\left(\left|R_{p}^{n-1}\right|_{1}+\left|R_{p}^{n-1}\right|_{0}\right) \\
& =\left|R_{p}^{n-1}\right|_{0}+\frac{p-1}{2}\left|R_{p}^{n-1}\right| \\
& =\left|R_{p}^{n-1}\right|_{0}+\frac{p-1}{2}\left(p^{n-1}-1\right) \\
& =\left|R_{p}^{n-1}\right|_{0}+\frac{1}{2}\left(p^{n}-p^{n-1}-p+1\right) .
\end{aligned}
$$

Recursively, we obtain from (2.14) that

$$
\begin{aligned}
\left|R_{p}^{n}\right|_{0}-\left|R_{p}^{n-1}\right|_{0} & =\frac{1}{2}\left(p^{n}-p^{n-1}-p+1\right) \\
\left|R_{p}^{n-1}\right|_{0}-\left|R_{p}^{n-2}\right|_{0} & =\frac{1}{2}\left(p^{n-1}-p^{n-2}-p+1\right)
\end{aligned}
$$

$$
\left|R_{p}^{1}\right|_{0}-\left|R_{p}^{0}\right|_{0}=\frac{1}{2}\left(p^{1}-p^{0}-p+1\right) .
$$

Note that $\left|R_{p}^{0}\right|_{0}=0$. By adding all left terms and all right terms of (2.15), respectively, we get

$$
\left|R_{p}^{n}\right|_{0}=\left|R_{p}^{n}\right|_{0}-\left|R_{p}^{0}\right|_{0}=\frac{1}{2}\left(p^{n}-n p+n-1\right) .
$$


Since the number of 1 s can be computed by subtracting the number of 0 s from the total number of creases, we have

$$
\begin{aligned}
\left|R_{p}^{n}\right|_{1} & =\left|R_{p}^{n}\right|-\left|R_{p}^{n}\right|_{0} \\
& =\left(p^{n}-1\right)-\frac{1}{2}\left(p^{n}-n p+n-1\right) \\
& =\frac{1}{2}\left(p^{n}+n p-n-1\right) .
\end{aligned}
$$

Thus we complete the proof.

\section{Number of 0 s and $1 \mathrm{~s}$ in $\left(R_{p} R_{q}\right)^{n}$}

In this section, we compute the number of $0 \mathrm{~s}$ and $1 \mathrm{~s}$ in $\left(R_{p} R_{q}\right)^{n}$. First, we estimate the number of $0 \mathrm{~s}$ and $1 \mathrm{~s}$ in $\left(R_{p} R_{q}\right)^{n}$ when $p$ and $q$ are even.

Theorem 3.1. Let $p$ and $q$ be even numbers with $p \geq 2$ and $q \geq 2$. For $n \in \mathbb{N}$, we have

$$
\left|\left(R_{p} R_{q}\right)^{n}\right|_{0}=\frac{1}{2}\left((p q)^{n}-p\right) \quad \text { and } \quad\left|\left(R_{p} R_{q}\right)^{n}\right|_{1}=\frac{1}{2}\left((p q)^{n}+p-2\right) .
$$

Proof. Since $p$ and $q$ are even, Theorem 2.1 gives

$$
\begin{aligned}
& \left(R_{p} R_{q}\right)^{n} \\
(3.2)= & R_{p}\left(R_{q}\left(R_{p} R_{q}\right)^{n-1}\right) \\
= & \left(\left(R_{q}\left(R_{p} R_{q}\right)^{n-1}\right)^{c} 1 R_{q}\left(R_{p} R_{q}\right)^{n-1} 1 \cdots 1 R_{q}\left(R_{p} R_{q}\right)^{n-1}\right)
\end{aligned}
$$

and

$$
\begin{aligned}
& R_{q}\left(R_{p} R_{q}\right)^{n-1} \\
= & R_{q}\left(\left(R_{p} R_{q}\right)^{n-1}\right) \\
= & \left(\left(\left(R_{p} R_{q}\right)^{n-1}\right)^{c} 1\left(R_{p} R_{q}\right)^{n-1} 1 \cdots 1\left(R_{p} R_{q}\right)^{n-1}\right) .
\end{aligned}
$$

$\left(R_{q}\left(R_{p} R_{q}\right)^{n-1}\right)^{c}$ and $R_{q}\left(R_{p} R_{q}\right)^{n-1}$ appear $\frac{p}{2}$ times and $\frac{p}{2}$ times, respectively, and 1 appears $p-1$ times in (3.2). In addition, $\left(\left(R_{p} R_{q}\right)^{n-1}\right)^{c}$ and $\left(R_{p} R_{q}\right)^{n-1}$ appear $\frac{q}{2}$ times and $\frac{q}{2}$ times, respectively, and 1 appears $q-1$ times in (3.3). By (3.2), (3.3) and Lemma 1.2, we have

$$
\begin{aligned}
\left|\left(R_{p} R_{q}\right)^{n}\right| & =\frac{p}{2}\left|\left(R_{q}\left(R_{p} R_{q}\right)^{n-1}\right)^{c}\right|+\frac{p}{2}\left|R_{q}\left(R_{p} R_{q}\right)^{n-1}\right|+(p-1) \\
& =\frac{p}{2}\left|R_{q}\left(R_{p} R_{q}\right)^{n-1}\right|+\frac{p}{2}\left|R_{q}\left(R_{p} R_{q}\right)^{n-1}\right|+(p-1) \\
& =p\left|R_{q}\left(R_{p} R_{q}\right)^{n-1}\right|+(p-1)
\end{aligned}
$$


and

$$
\begin{aligned}
\left|R_{q}\left(R_{p} R_{q}\right)^{n-1}\right| & =\frac{q}{2}\left|\left(\left(R_{p} R_{q}\right)^{n-1}\right)^{c}\right|+\frac{q}{2}\left|\left(R_{p} R_{q}\right)^{n-1}\right|+(q-1) \\
& =\frac{q}{2}\left|\left(R_{p} R_{q}\right)^{n-1}\right|+\frac{q}{2}\left|\left(R_{p} R_{q}\right)^{n-1}\right|+(q-1) \\
& =q\left|\left(R_{p} R_{q}\right)^{n-1}\right|+(q-1) .
\end{aligned}
$$

From (3.4) and (3.5), we get

$$
\begin{aligned}
\left|\left(R_{p} R_{q}\right)^{n}\right| & =p\left|R_{q}\left(R_{p} R_{q}\right)^{n-1}\right|+(p-1) \\
& =p\left(q\left|\left(R_{p} R_{q}\right)^{n-1}\right|+(q-1)\right)+(p-1) \\
& =p q\left|\left(R_{p} R_{q}\right)^{n-1}\right|+p q-1 .
\end{aligned}
$$

By adding 1 on both sides of (3.6), we have

$$
\begin{aligned}
\left|\left(R_{p} R_{q}\right)^{n}\right|+1 & =p q\left|\left(R_{p} R_{q}\right)^{n-1}\right|+p q \\
& =p q\left(\left|\left(R_{p} R_{q}\right)^{n-1}\right|+1\right) \\
& =(p q)^{2}\left(\left|\left(R_{p} R_{q}\right)^{n-2}\right|+1\right) \\
& =\cdots \\
& =(p q)^{n}\left(\left|\left(R_{p} R_{q}\right)^{0}\right|+1\right) \\
& =(p q)^{n},
\end{aligned}
$$

since $\left|\left(R_{p} R_{q}\right)^{0}\right|=0$. Thus

$$
\left|\left(R_{p} R_{q}\right)^{n}\right|=(p q)^{n}-1
$$

and

$$
\begin{aligned}
\left|R_{q}\left(R_{p} R_{q}\right)^{n-1}\right| & =q\left|\left(R_{p} R_{q}\right)^{n-1}\right|+(q-1) \\
& =q\left((p q)^{n-1}-1\right)+(q-1) \\
& =p^{n-1} q^{n}-1 .
\end{aligned}
$$

Now, we compute the number of $0 \mathrm{~s}$ and $1 \mathrm{~s}$ in $\left(R_{p} R_{q}\right)^{n}$.

By (3.4), (3.9) and Lemma 1.2, we have

$$
\begin{aligned}
\left|\left(R_{p} R_{q}\right)^{n}\right|_{0} & =\frac{p}{2}\left|\left(R_{q}\left(R_{p} R_{q}\right)^{n-1}\right)^{c}\right|_{0}+\frac{p}{2}\left|R_{q}\left(R_{p} R_{q}\right)^{n-1}\right|_{0} \\
& =\frac{p}{2}\left|R_{q}\left(R_{p} R_{q}\right)^{n-1}\right|_{1}+\frac{p}{2}\left|R_{q}\left(R_{p} R_{q}\right)^{n-1}\right|_{0} \\
& =\frac{p}{2}\left|R_{q}\left(R_{p} R_{q}\right)^{n-1}\right| \\
& =\frac{p}{2}\left(p^{n-1} q^{n}-1\right) \\
& =\frac{1}{2}\left((p q)^{n}-p\right) .
\end{aligned}
$$


By (3.8) and (3.10), we finally have

$$
\begin{aligned}
\left|\left(R_{p} R_{q}\right)^{n}\right|_{1} & =\left|\left(R_{p} R_{q}\right)^{n}\right|-\left|\left(R_{p} R_{q}\right)^{n}\right|_{0} \\
& =\left((p q)^{n}-1\right)-\frac{1}{2}\left((p q)^{n}-p\right) \\
& =\frac{1}{2}\left((p q)^{n}+p-2\right) .
\end{aligned}
$$

Therefore we prove (3.1).

Now, we estimate the number of $0 \mathrm{~s}$ and $1 \mathrm{~s}$ in $\left(R_{p} R_{q}\right)^{n}$ when $p$ is even and $q$ is odd.

Theorem 3.2. Let $p$ be an even number with $p \geq 2$ and let $q$ be an odd number with $q \geq 3$. For $n \in \mathbb{N}$, we have

$$
\left|\left(R_{p} R_{q}\right)^{n}\right|_{0}=\frac{1}{2}\left((p q)^{n}-p\right) \quad \text { and } \quad\left|\left(R_{p} R_{q}\right)^{n}\right|_{1}=\frac{1}{2}\left((p q)^{n}+p-2\right) .
$$

Proof. Since $p$ is even and $q$ is odd, Theorem 2.1 gives

$$
\begin{aligned}
& \left(R_{p} R_{q}\right)^{n} \\
(3.13)= & R_{p}\left(R_{q}\left(R_{p} R_{q}\right)^{n-1}\right) \\
= & \left(\left(R_{q}\left(R_{p} R_{q}\right)^{n-1}\right)^{c} 1 R_{q}\left(R_{p} R_{q}\right)^{n-1} 1 \cdots 1 R_{q}\left(R_{p} R_{q}\right)^{n-1}\right)
\end{aligned}
$$

and

$$
\begin{aligned}
& R_{q}\left(R_{p} R_{q}\right)^{n-1} \\
= & R_{q}\left(\left(R_{p} R_{q}\right)^{n-1}\right) \\
= & \left(\left(R_{p} R_{q}\right)^{n-1} 1\left(\left(R_{p} R_{q}\right)^{n-1}\right)^{c} 1 \cdots 1\left(R_{p} R_{q}\right)^{n-1}\right) .
\end{aligned}
$$

$\left(R_{q}\left(R_{p} R_{q}\right)^{n-1}\right)^{c}$ and $R_{q}\left(R_{p} R_{q}\right)^{n-1}$ appear $\frac{p}{2}$ times and $\frac{p}{2}$ times, respectively, and 1 appears $p-1$ times in (3.13). In addition, $\left(\left(R_{p} R_{q}\right)^{n-1}\right)^{c}$ and $\left(R_{p} R_{q}\right)^{n-1}$ appear $\frac{q-1}{2}$ times and $\frac{q+1}{2}$ times, respectively, and 1 appears $q-1$ times in (3.14). By (3.13), (3.14) and Lemma 1.2, we have

$$
\begin{aligned}
\left|\left(R_{p} R_{q}\right)^{n}\right| & =\frac{p}{2}\left|\left(R_{q}\left(R_{p} R_{q}\right)^{n-1}\right)^{c}\right|+\frac{p}{2}\left|R_{q}\left(R_{p} R_{q}\right)^{n-1}\right|+(p-1) \\
& =\frac{p}{2}\left|R_{q}\left(R_{p} R_{q}\right)^{n-1}\right|+\frac{p}{2}\left|R_{q}\left(R_{p} R_{q}\right)^{n-1}\right|+(p-1) \\
& =p\left|R_{q}\left(R_{p} R_{q}\right)^{n-1}\right|+(p-1)
\end{aligned}
$$


and

$$
\begin{aligned}
\left|R_{q}\left(R_{p} R_{q}\right)^{n-1}\right| & =\frac{q-1}{2}\left|\left(\left(R_{p} R_{q}\right)^{n-1}\right)^{c}\right|+\frac{q+1}{2}\left|\left(R_{p} R_{q}\right)^{n-1}\right|+(q-1) \\
(3.16) & =\frac{q-1}{2}\left|\left(R_{p} R_{q}\right)^{n-1}\right|+\frac{q+1}{2}\left|\left(R_{p} R_{q}\right)^{n-1}\right|+(q-1) \\
& =q\left|\left(R_{p} R_{q}\right)^{n-1}\right|+(q-1) .
\end{aligned}
$$

From (3.15) and (3.16), we get

$$
\begin{aligned}
\left|\left(R_{p} R_{q}\right)^{n}\right| & =p\left|R_{q}\left(R_{p} R_{q}\right)^{n-1}\right|+(p-1) \\
& =p\left(q\left|\left(R_{p} R_{q}\right)^{n-1}\right|+(q-1)\right)+(p-1) \\
& =p q\left|\left(R_{p} R_{q}\right)^{n-1}\right|+p q-1 .
\end{aligned}
$$

By adding 1 on both sides of (3.17), we have

$$
\begin{aligned}
\left|\left(R_{p} R_{q}\right)^{n}\right|+1 & =p q\left|\left(R_{p} R_{q}\right)^{n-1}\right|+p q \\
& =p q\left(\left|\left(R_{p} R_{q}\right)^{n-1}\right|+1\right) \\
& =(p q)^{2}\left(\left|\left(R_{p} R_{q}\right)^{n-2}\right|+1\right) \\
& =\cdots \\
& =(p q)^{n}\left(\left|\left(R_{p} R_{q}\right)^{0}\right|+1\right) \\
& =(p q)^{n},
\end{aligned}
$$

since $\left|\left(R_{p} R_{q}\right)^{0}\right|=0$. Thus

$$
\left|\left(R_{p} R_{q}\right)^{n}\right|=(p q)^{n}-1
$$

and

$$
\begin{aligned}
\left|R_{q}\left(R_{p} R_{q}\right)^{n-1}\right| & =q\left|\left(R_{p} R_{q}\right)^{n-1}\right|+(q-1) \\
& =q\left((p q)^{n-1}-1\right)+(q-1) \\
& =p^{n-1} q^{n}-1 .
\end{aligned}
$$

Now, we compute the number of $0 \mathrm{~s}$ and $1 \mathrm{~s}$ in $\left(R_{p} R_{q}\right)^{n}$.

By (3.15), (3.20) and Lemma 1.2, we have

$$
\begin{aligned}
\left|\left(R_{p} R_{q}\right)^{n}\right|_{0} & =\frac{p}{2}\left|\left(R_{q}\left(R_{p} R_{q}\right)^{n-1}\right)^{c}\right|_{0}+\frac{p}{2}\left|R_{q}\left(R_{p} R_{q}\right)^{n-1}\right|_{0} \\
& =\frac{p}{2}\left|\left(R_{q}\left(R_{p} R_{q}\right)^{n-1}\right)\right|_{1}+\frac{p}{2}\left|R_{q}\left(R_{p} R_{q}\right)^{n-1}\right|_{0} \\
& =\frac{p}{2}\left|R_{q}\left(R_{p} R_{q}\right)^{n-1}\right| \\
& =\frac{p}{2}\left(p^{n-1} q^{n}-1\right) \\
& =\frac{1}{2}\left((p q)^{n}-p\right) .
\end{aligned}
$$


By (3.19) and (3.21), we finally have

$$
\begin{aligned}
\left|\left(R_{p} R_{q}\right)^{n}\right|_{1} & =\left|\left(R_{p} R_{q}\right)^{n}\right|-\left|\left(R_{p} R_{q}\right)^{n}\right|_{0} \\
& =\left((p q)^{n}-1\right)-\frac{1}{2}\left((p q)^{n}-p\right) \\
& =\frac{1}{2}\left((p q)^{n}+p-2\right) .
\end{aligned}
$$

Therefore we prove (3.12).

Now, we estimate the number of $0 \mathrm{~s}$ and $1 \mathrm{~s}$ in $\left(R_{p} R_{q}\right)^{n}$ when $p$ is odd and $q$ is even.

Theorem 3.3. Let $p$ be an odd number with $p \geq 3$ and let $q$ be an even number with $q \geq 2$. For $n \in \mathbb{N}$, we have

$$
\left|\left(R_{p} R_{q}\right)^{n}\right|_{0}=\frac{1}{2}\left((p q)^{n}-p-q+1\right)
$$

and

$$
\left|\left(R_{p} R_{q}\right)^{n}\right|_{1}=\frac{1}{2}\left((p q)^{n}+p+q-3\right) .
$$

Proof. Since $p$ is odd and $q$ is even, Theorem 2.1 gives

$$
\begin{aligned}
& \left(R_{p} R_{q}\right)^{n} \\
(3.25)= & R_{p}\left(R_{q}\left(R_{p} R_{q}\right)^{n-1}\right) \\
= & \left(R_{q}\left(R_{p} R_{q}\right)^{n-1} 1\left(R_{q}\left(R_{p} R_{q}\right)^{n-1}\right)^{c} 1 \cdots 1 R_{q}\left(R_{p} R_{q}\right)^{n-1}\right)
\end{aligned}
$$

and

$$
\begin{aligned}
& R_{q}\left(R_{p} R_{q}\right)^{n-1} \\
= & R_{q}\left(\left(R_{p} R_{q}\right)^{n-1}\right) \\
= & \left(\left(\left(R_{p} R_{q}\right)^{n-1}\right)^{c} 1\left(R_{p} R_{q}\right)^{n-1} 1 \cdots 1\left(R_{p} R_{q}\right)^{n-1}\right) .
\end{aligned}
$$

$\left(R_{q}\left(R_{p} R_{q}\right)^{n-1}\right)^{c}$ and $R_{q}\left(R_{p} R_{q}\right)^{n-1}$ appear $\frac{p-1}{2}$ times and $\frac{p+1}{2}$ times, respectively, and 1 appears $p-1$ times in (3.25). In addition, $\left(\left(R_{p} R_{q}\right)^{n-1}\right)^{c}$ and $\left(R_{p} R_{q}\right)^{n-1}$ appear $\frac{q}{2}$ times and $\frac{q}{2}$ times, respectively, and 1 appears $q-1$ times in (3.26). By (3.25), (3.26) and Lemma 1.2, we have

$$
\begin{aligned}
\left|\left(R_{p} R_{q}\right)^{n}\right| & =\frac{p-1}{2}\left|\left(R_{q}\left(R_{p} R_{q}\right)^{n-1}\right)^{c}\right|+\frac{p+1}{2}\left|R_{q}\left(R_{p} R_{q}\right)^{n-1}\right|+(p-1) \\
(3.27) & =\frac{p-1}{2}\left|R_{q}\left(R_{p} R_{q}\right)^{n-1}\right|+\frac{p+1}{2}\left|R_{q}\left(R_{p} R_{q}\right)^{n-1}\right|+(p-1) \\
& =p\left|R_{q}\left(R_{p} R_{q}\right)^{n-1}\right|+(p-1)
\end{aligned}
$$


and

$$
\begin{aligned}
\left|R_{q}\left(R_{p} R_{q}\right)^{n-1}\right| & =\frac{q}{2}\left|\left(\left(R_{p} R_{q}\right)^{n-1}\right)^{c}\right|+\frac{q}{2}\left|\left(R_{p} R_{q}\right)^{n-1}\right|+(q-1) \\
& =\frac{q}{2}\left|\left(R_{p} R_{q}\right)^{n-1}\right|+\frac{q}{2}\left|\left(R_{p} R_{q}\right)^{n-1}\right|+(q-1) \\
& =q\left|\left(R_{p} R_{q}\right)^{n-1}\right|+(q-1) .
\end{aligned}
$$

From (3.27) and (3.28), we get

$$
\begin{aligned}
\left|\left(R_{p} R_{q}\right)^{n}\right| & =p\left|R_{q}\left(R_{p} R_{q}\right)^{n-1}\right|+(p-1) \\
& =p\left(q\left|\left(R_{p} R_{q}\right)^{n-1}\right|+(q-1)\right)+(p-1) \\
& =p q\left|\left(R_{p} R_{q}\right)^{n-1}\right|+p q-1 .
\end{aligned}
$$

By adding 1 on both sides of (3.29), we have

$$
\begin{aligned}
\left|\left(R_{p} R_{q}\right)^{n}\right|+1 & =p q\left|\left(R_{p} R_{q}\right)^{n-1}\right|+p q \\
& =p q\left(\left|\left(R_{p} R_{q}\right)^{n-1}\right|+1\right) \\
& =(p q)^{2}\left(\left|\left(R_{p} R_{q}\right)^{n-2}\right|+1\right) \\
& =\cdots \\
& =(p q)^{n}\left(\left|\left(R_{p} R_{q}\right)^{0}\right|+1\right) \\
& =(p q)^{n},
\end{aligned}
$$

since $\left|\left(R_{p} R_{q}\right)^{0}\right|=0$. Thus

$$
\left|\left(R_{p} R_{q}\right)^{n}\right|=(p q)^{n}-1
$$

and

$$
\begin{aligned}
\left|R_{q}\left(R_{p} R_{q}\right)^{n-1}\right| & =q\left|\left(R_{p} R_{q}\right)^{n-1}\right|+(q-1) \\
& =q\left((p q)^{n-1}-1\right)+(q-1) \\
& =p^{n-1} q^{n}-1 .
\end{aligned}
$$

Now, we compute the number of $0 \mathrm{~s}$ and $1 \mathrm{~s}$ in $\left(R_{p} R_{q}\right)^{n}$. 
By (3.27), (3.28), (3.31), (3.32) and Lemma 1.2, we have

$$
\text { (3.33) } \begin{aligned}
& \left|\left(R_{p} R_{q}\right)^{n}\right|_{0} \\
= & \frac{p-1}{2}\left|\left(R_{q}\left(R_{p} R_{q}\right)^{n-1}\right)^{c}\right|_{0}+\frac{p+1}{2}\left|R_{q}\left(R_{p} R_{q}\right)^{n-1}\right|_{0} \\
= & \frac{p-1}{2}\left|R_{q}\left(R_{p} R_{q}\right)^{n-1}\right|_{1}+\frac{p+1}{2}\left|R_{q}\left(R_{p} R_{q}\right)^{n-1}\right|_{0} \\
= & \left|R_{q}\left(R_{p} R_{q}\right)^{n-1}\right|_{0}+\frac{p-1}{2}\left(\left|R_{q}\left(R_{p} R_{q}\right)^{n-1}\right|_{1}+\left|R_{q}\left(R_{p} R_{q}\right)^{n-1}\right|_{0}\right) \\
= & \left|R_{q}\left(R_{p} R_{q}\right)^{n-1}\right|_{0}+\frac{p-1}{2}\left|R_{q}\left(R_{p} R_{q}\right)^{n-1}\right| \\
= & \frac{q}{2}\left(\left|\left(\left(R_{p} R_{q}\right)^{n-1}\right)^{c}\right|_{0}+\left|\left(R_{p} R_{q}\right)^{n-1}\right|_{0}\right)+\frac{p-1}{2}\left|R_{q}\left(R_{p} R_{q}\right)^{n-1}\right| \\
= & \frac{q}{2}\left(\left|\left(R_{p} R_{q}\right)^{n-1}\right|_{1}+\left|\left(R_{p} R_{q}\right)^{n-1}\right|_{0}\right)+\frac{p-1}{2}\left|R_{q}\left(R_{p} R_{q}\right)^{n-1}\right| \\
= & \frac{q}{2}\left|\left(R_{p} R_{q}\right)^{n-1}\right|+\frac{p-1}{2}\left|R_{q}\left(R_{p} R_{q}\right)^{n-1}\right| \\
= & \frac{q}{2}\left(p^{n-1} q^{n-1}-1\right)+\frac{p-1}{2}\left(p^{n-1} q^{n}-1\right) \\
= & \frac{1}{2}\left((p q)^{n}-p-q+1\right) .
\end{aligned}
$$

By (3.31) and (3.33), we finally have

$$
\begin{aligned}
\left|\left(R_{p} R_{q}\right)^{n}\right|_{1} & =\left|\left(R_{p} R_{q}\right)^{n}\right|-\left|\left(R_{p} R_{q}\right)^{n}\right|_{0} \\
& =\left((p q)^{n}-1\right)-\frac{1}{2}\left((p q)^{n}-p-q+1\right) \\
& =\frac{1}{2}\left((p q)^{n}+p+q-3\right) .
\end{aligned}
$$

Therefore we prove (3.23) and (3.24).

Finally, we estimate the number of 0 s and $1 \mathrm{~s}$ in $\left(R_{p} R_{q}\right)^{n}$ when $p$ and $q$ are odd. In the proof, we use special properties of recursive sequences that are not used in Theorem 3.1, Theorem 3.2 and Theorem 3.3.

Theorem 3.4. Let $p$ and $q$ be odd numbers with $p \geq 3$ and $q \geq 3$. For $n \in \mathbb{N}$, we have

$$
\left|\left(R_{p} R_{q}\right)^{n}\right|_{0}=\frac{1}{2}\left((p q)^{n}-n(p+q-2)-1\right)
$$

and

$$
\left|\left(R_{p} R_{q}\right)^{n}\right|_{1}=\frac{1}{2}\left((p q)^{n}+n(p+q-2)-1\right) .
$$


Proof. Since $p$ and $q$ are odd, Theorem 2.1 gives

$$
\begin{aligned}
& \left(R_{p} R_{q}\right)^{n} \\
(3.37)= & R_{p}\left(R_{q}\left(R_{p} R_{q}\right)^{n-1}\right) \\
= & \left(R_{q}\left(R_{p} R_{q}\right)^{n-1} 1\left(R_{q}\left(R_{p} R_{q}\right)^{n-1}\right)^{c} 1 \cdots 1 R_{q}\left(R_{p} R_{q}\right)^{n-1}\right)
\end{aligned}
$$

and

$$
\begin{aligned}
& R_{q}\left(R_{p} R_{q}\right)^{n-1} \\
= & R_{q}\left(\left(R_{p} R_{q}\right)^{n-1}\right) \\
= & \left(\left(R_{p} R_{q}\right)^{n-1} 1\left(\left(R_{p} R_{q}\right)^{n-1}\right)^{c} 1 \cdots 1\left(R_{p} R_{q}\right)^{n-1}\right) .
\end{aligned}
$$

$\left(R_{q}\left(R_{p} R_{q}\right)^{n-1}\right)^{c}$ and $R_{q}\left(R_{p} R_{q}\right)^{n-1}$ appear $\frac{p-1}{2}$ times and $\frac{p+1}{2}$ times, respectively, and 1 appears $p-1$ times in (3.37). In addition, $\left(\left(R_{p} R_{q}\right)^{n-1}\right)^{c}$ and $\left(R_{p} R_{q}\right)^{n-1}$ appear $\frac{q-1}{2}$ times and $\frac{q+1}{2}$ times, respectively, and 1 appears $q-1$ times in (3.38). By (3.37), (3.38) and Lemma 1.2, we have

$$
\begin{aligned}
\left|\left(R_{p} R_{q}\right)^{n}\right| & =\frac{p-1}{2}\left|\left(R_{q}\left(R_{p} R_{q}\right)^{n-1}\right)^{c}\right|+\frac{p+1}{2}\left|R_{q}\left(R_{p} R_{q}\right)^{n-1}\right|+(p-1) \\
(3.39) & =\frac{p-1}{2}\left|R_{q}\left(R_{p} R_{q}\right)^{n-1}\right|+\frac{p+1}{2}\left|R_{q}\left(R_{p} R_{q}\right)^{n-1}\right|+(p-1) \\
& =p\left|R_{q}\left(R_{p} R_{q}\right)^{n-1}\right|+(p-1)
\end{aligned}
$$

and

$$
\begin{aligned}
\left|R_{q}\left(R_{p} R_{q}\right)^{n-1}\right| & =\frac{q-1}{2}\left|\left(\left(R_{p} R_{q}\right)^{n-1}\right)^{c}\right|+\frac{q+1}{2}\left|\left(R_{p} R_{q}\right)^{n-1}\right|+(q-1) \\
(3.40) & =\frac{q-1}{2}\left|\left(R_{p} R_{q}\right)^{n-1}\right|+\frac{q+1}{2}\left|\left(R_{p} R_{q}\right)^{n-1}\right|+(q-1) \\
& =q\left|\left(R_{p} R_{q}\right)^{n-1}\right|+(q-1) .
\end{aligned}
$$

From (3.39) and (3.40), we get

$$
\begin{aligned}
\left|\left(R_{p} R_{q}\right)^{n}\right| & =p\left|R_{q}\left(R_{p} R_{q}\right)^{n-1}\right|+(p-1) \\
& =p\left(q\left|\left(R_{p} R_{q}\right)^{n-1}\right|+(q-1)\right)+(p-1) \\
& =p q\left|\left(R_{p} R_{q}\right)^{n-1}\right|+p q-1 .
\end{aligned}
$$


By adding 1 on both sides of (3.41), we have

$$
\begin{aligned}
\left|\left(R_{p} R_{q}\right)^{n}\right|+1 & =p q\left|\left(R_{p} R_{q}\right)^{n-1}\right|+p q \\
& =p q\left(\left|\left(R_{p} R_{q}\right)^{n-1}\right|+1\right) \\
& =(p q)^{2}\left(\left|\left(R_{p} R_{q}\right)^{n-2}\right|+1\right) \\
& =\cdots \\
& =(p q)^{n}\left(\left|\left(R_{p} R_{q}\right)^{0}\right|+1\right) \\
& =(p q)^{n},
\end{aligned}
$$

since $\left|\left(R_{p} R_{q}\right)^{0}\right|=0$. Thus

$$
\left|\left(R_{p} R_{q}\right)^{n}\right|=(p q)^{n}-1
$$

and

$$
\begin{aligned}
\left|R_{q}\left(R_{p} R_{q}\right)^{n-1}\right| & =q\left|\left(R_{p} R_{q}\right)^{n-1}\right|+(q-1) \\
& =q\left((p q)^{n-1}-1\right)+(q-1) \\
& =p^{n-1} q^{n}-1 .
\end{aligned}
$$

Now, we compute the number of $0 \mathrm{~s}$ and $1 \mathrm{~s}$ in $\left(R_{p} R_{q}\right)^{n}$.

By (3.39) and Lemma 1.2, we have

$$
\begin{aligned}
& \left|\left(R_{p} R_{q}\right)^{n}\right|_{0} \\
= & \frac{p-1}{2}\left|\left(R_{q}\left(R_{p} R_{q}\right)^{n-1}\right)^{c}\right|_{0}+\frac{p+1}{2}\left|R_{q}\left(R_{p} R_{q}\right)^{n-1}\right|_{0} \\
= & \frac{p-1}{2}\left|R_{q}\left(R_{p} R_{q}\right)^{n-1}\right|_{1}+\frac{p+1}{2}\left|R_{q}\left(R_{p} R_{q}\right)^{n-1}\right|_{0}
\end{aligned}
$$

and

$$
\begin{aligned}
& =\frac{p-1}{2}\left|\left(R_{q}\left(R_{p} R_{q}\right)^{n-1}\right)^{c}\right|_{1}+\frac{p+1}{2}\left|R_{q}\left(R_{p} R_{q}\right)^{n-1}\right|_{1}+(p-1) \\
& =\frac{p-1}{2}\left|R_{q}\left(R_{p} R_{q}\right)^{n-1}\right|_{0}+\frac{p+1}{2}\left|R_{q}\left(R_{p} R_{q}\right)^{n-1}\right|_{1}+(p-1) .
\end{aligned}
$$

From (3.45) and (3.46), we get

$$
\begin{aligned}
& \left|\left(R_{p} R_{q}\right)^{n}\right|_{1}-\left|\left(R_{p} R_{q}\right)^{n}\right|_{0} \\
= & \left|R_{q}\left(R_{p} R_{q}\right)^{n-1}\right|_{1}-\left|R_{q}\left(R_{p} R_{q}\right)^{n-1}\right|_{0}+(p-1) .
\end{aligned}
$$


By (3.40) and Lemma 1.2, we have

$$
\begin{aligned}
& \left|R_{q}\left(R_{p} R_{q}\right)^{n-1}\right|_{0} \\
= & \frac{q-1}{2}\left|\left(\left(R_{p} R_{q}\right)^{n-1}\right)^{c}\right|_{0}+\frac{q+1}{2}\left|\left(R_{p} R_{q}\right)^{n-1}\right|_{0} \\
= & \frac{q-1}{2}\left|\left(R_{p} R_{q}\right)^{n-1}\right|_{1}+\frac{q+1}{2}\left|\left(R_{p} R_{q}\right)^{n-1}\right|_{0}
\end{aligned}
$$

and

$$
\begin{aligned}
& \left|R_{q}\left(R_{p} R_{q}\right)^{n-1}\right|_{1} \\
= & \frac{q-1}{2}\left|\left(\left(R_{p} R_{q}\right)^{n-1}\right)^{c}\right|_{1}+\frac{q+1}{2}\left|\left(R_{p} R_{q}\right)^{n-1}\right|_{1}+(q-1) \\
= & \frac{q-1}{2}\left|\left(R_{p} R_{q}\right)^{n-1}\right|_{0}+\frac{q+1}{2}\left|\left(R_{p} R_{q}\right)^{n-1}\right|_{1}+(q-1) .
\end{aligned}
$$

From (3.48) and (3.49), we get

$$
\begin{aligned}
& \left|R_{q}\left(R_{p} R_{q}\right)^{n-1}\right|_{1}-\left|R_{q}\left(R_{p} R_{q}\right)^{n-1}\right|_{0} \\
= & \left|\left(R_{p} R_{q}\right)^{n-1}\right|_{1}-\left|\left(R_{p} R_{q}\right)^{n-1}\right|_{0}+(q-1) .
\end{aligned}
$$

By (3.47) and (3.50), we get

$$
\begin{aligned}
& \left|\left(R_{p} R_{q}\right)^{n}\right|_{1}-\left|\left(R_{p} R_{q}\right)^{n}\right|_{0} \\
= & \left|\left(R_{p} R_{q}\right)^{n-1}\right|_{1}-\left|\left(R_{p} R_{q}\right)^{n-1}\right|_{0}+(p-1)+(q-1) \\
= & \left|\left(R_{p} R_{q}\right)^{n-2}\right|_{1}-\left|\left(R_{p} R_{q}\right)^{n-2}\right|_{0}+2(p-1)+2(q-1) \\
= & \cdots \\
= & \left|\left(R_{p} R_{q}\right)^{0}\right|_{1}-\left|\left(R_{p} R_{q}\right)^{0}\right|_{0}+n(p-1)+n(q-1) .
\end{aligned}
$$

Since $\left|\left(R_{p} R_{q}\right)^{0}\right|_{1}=\left|\left(R_{p} R_{q}\right)^{0}\right|_{0}=0$, we get

$$
\begin{aligned}
\left|\left(R_{p} R_{q}\right)^{n}\right|_{1}-\left|\left(R_{p} R_{q}\right)^{n}\right|_{0} & =n(p-1)+n(q-1) \\
& =n(p+q-2) .
\end{aligned}
$$

From (3.43) and Lemma 1.2, we have

$$
\left|\left(R_{p} R_{q}\right)^{n}\right|_{1}+\left|\left(R_{p} R_{q}\right)^{n}\right|_{0}=\left|\left(R_{p} R_{q}\right)^{n}\right|=(p q)^{n}-1 .
$$

By combining (3.52) and (3.53), we have

$$
\left|\left(R_{p} R_{q}\right)^{n}\right|_{0}=\frac{1}{2}\left((p q)^{n}-n(p+q-2)-1\right)
$$

and

$$
\left|\left(R_{p} R_{q}\right)^{n}\right|_{1}=\frac{1}{2}\left((p q)^{n}+n(p+q-2)-1\right) .
$$

Therefore we prove (3.35) and (3.36). 
From Theorem 3.1, Theorem 3.2, Theorem 3.3 and Theorem 3.4, we obtain the following.

Corollary 3.5. For any $p, q \in \mathbb{N}$ with $p \geq 2$ and $q \geq 2$, we have

$$
\left|\left(R_{p} R_{q}\right)^{n}\right|=(p q)^{n}-1 .
$$

\section{Acknowledgements}

The authors wish to express their gratitude to Sooji Hwang, Jinrae Kim, Sunggye Lee, Chansu Park, Jeongyin Park(2nd grade) and Junhwi Lim(1st grade) at Incheon Science High School for their valuable comments and discussion which was a major contribution to this research.

\section{References}

[1] Jean-Paul Allouche, The number of factors in a paperfolding sequence, Bull. Austral. Math. Soc., 46 (1992), 23-32.

[2] Bruce Bates, Martin Bunder and Keith Tognetti, Mirroring and interleaving in the paperfolding sequence, Appl. Anal. Discrete Math., 4 (2010), 96-118.

[3] Chandler Davis and Donald E. Knuth, Number representations and dragon curves, J. Rec. Math., 3 (1970), 66-81.

[4] Michel Dekking, Paperfolding, morphisms, planefilling, curves and fractal tiles, Theor. Comput. Sci., 414(1) (2012), 20-37

[5] M. Mendes France and A. J. Van Der Poorten, Arithmetic and analytic properties of paper folding sequences, Bull. Austral. Math. Soc. 24(1981), 123-131.

[6] Sunggye Lee, Jinsoo Kim and Won Choi, Relation between folding and unfolding paper of rectangle and (0,1)-pattern, J. Korean Soc. Math. Ed. Ser. E, 23(3)(2009), 507-522.

Junghee Yun

Incheon Science High School,

Incheon 400-831, Korea.

E-mail: matheducation@hanmail.net

Nahmwoo Hahm

Department of Mathematics, University of Incheon,

Incheon 406-772, Korea.

E-mail: nhahm@incheon.ac.kr 